\title{
DEGRADACIÓN FOTOCATALÍTICA DE AROCLOR 1254 UTILIZANDO DIÓXIDO DE TITANIO IRRADIADO CON HAZ DE ELECTRONES ACELERADOS
}

\author{
PHOTOCATALYTIC DEGRADATION OF AROCLOR 1254 \\ USING TITANIUM DIOXIDE IRRADIATED WITH ACCELERATED \\ ELECTRON BEAM
}

\begin{abstract}
Gabriel Almeida P. ${ }^{1,2}$, Lenys Fernández ${ }^{1,3}$, Ramiro Castro ${ }^{4}$, William Villacis², Patricio Espinoza-Montero ${ }^{1 *}$
\end{abstract}

Recibido: 1 de mayo 2019 / Aceptado: 20 de junio2019

DOI: $10.26807 /$ ia.v7i2.107

\begin{abstract}
Palabras clave: Electrones acelerados, fotocatálisis heterogénea, irradiación de $\mathrm{TiO}_{2}$, policloruros de bifenilo.

Keywords: Biphenyls polychlorides, electron beams, heterogeneous photocatalysis, $\mathrm{TiO}_{2}$ irradiation.
\end{abstract}

1 Pontificia Universidad Católica del Ecuador, Escuela de Ciencias Químicas, Quito, Ecuador. (gabriel.almeida@epn.edu.ec; Imfernandez@puce.edu.ec; *correspondencia: pespinoza646@puce.edu.ec)

2 Escuela Politécnica Nacional, Departamento de Ciencias Nucleares, Quito, Ecuador. (gabriel.al meida@epn.edu.ec; william.villacis@epn.edu.ec)

3 Universidad Simón Bolívar, Departamento de Química, Caracas, Venezuela. (Ifernandez@usb.ve)

4 Ministerio de Electricidad y Recursos Naturales no Renovables, Laboratorio de Análisis Químico de Contaminantes Orgánicos, Quito, Ecuador. (cesar.castro@meer.gob.ec) 


\section{RESUMEN}

Los policloruros de bifenilo ( $\mathrm{PCB}^{\prime} \mathrm{s}$ ) son compuestos orgánicos que fueron usados en condensadores y transformadores eléctricos debido a sus propiedades aislantes y alta estabilidad química. Al prohibirse la fabricación y el uso de PCB's, gran cantidad de estos equipos eléctricos quedaron obsoletos y enormes volúmenes de aceite dieléctrico con PCB's se almacenaron como desecho peligroso a la espera de una alternativa para su eliminación. En este trabajo se reporta la transferencia de fase del Aroclor 1254, desde el aceite dieléctrico hacia fase acuosa, para su posterior degradación fotocatalítica. Se probaron dos agentes de transferencia de fase, Polietilenglicol 400 y Tritón X-100. El proceso fotocatalítico se llevó a cabo con $\mathrm{TiO}_{2}$ sin irradiar (0 kGy) e irradiado con haz de electrones a dosis de 20 y 60 kGy, se utilizó radiación ultravioleta como fuente de energía durante la fotodegradación. La degradación se siguió por cromatografía de gases y la mineralización se verificó mediante Carbono Orgánico Total (COT). La mayor eficiencia de transferencia de fase se logró con el Tritón X100, por lo que se utilizó este agente en los estudios posteriores. Los mejores resultados de degradación y mineralización del Aroclor 1254, se alcanzó con el $\mathrm{TiO}_{2}$ irradiado a $20 \mathrm{kGy}$, estos fueron de $98,36 \%$ y $58,92 \%$ respectivamente, luego de 240 min de fotooxidación. De esta manera esta tecnología se convierte en una alternativa viable para solucionar el problema de la contaminación por PCBs.

\section{ABSTRACT}

Biphenyl polychlorides (PCB's) are organic compounds that were used in capacitors and electrical transformers due to their insulating properties and high chemical stability. By banning the manufacture and use of PCBs, a lot of this electrical equipment became obsolete and huge volumes of dielectric oil with PCBs were stored as hazardous waste pending an alternative for their treatment. In this work, the phase transfer of PCB's (Aroclor 1254) from the dielectric oil to an aqueous phase, for its subsequent photocatalytic degradation is proposed. Two transfer agents were probed, Polyethylene Glycol 400 and Triton X-100. The catalytic process was carried out with no irradiated $\mathrm{TiO}_{2}$ (0 kGy) and ebeam irradiated $\mathrm{TiO}_{2}$ with 20 and 60 kGy; UV-vis was used as a source of energy 
during photodegradation. The degradation was evaluated by gas chromatography and the mineralization was verified by Total Organic Carbon (COT). The highest phase transfer efficiency was achieved with the Triton X-100, so this agent was used in subsequent studies. The best results of degradation and mineralization of Aroclor 1254, was reached with the $\mathrm{TiO}_{2}$ irradiated at $20 \mathrm{kGy}$, these were $98.36 \%$ and $58.92 \%$ respectively, after 240 min of photo-oxidation. In this way this technology becomes a viable alternative to solve the problem of contamination by PCBs.

\section{INTRODUCCIÓN}

Los Policloruros de Bifenilo (PCB's) son compuestos orgánicos clorados sintéticos caracterizados por una baja reactividad y excelentes propiedades aislantes eléctricas y térmicas. Su principal aplicación es su uso como fluidos dieléctricos en capacitores y transformadores o en intercambiadores de calor (Huang \& Hong, 2000). Se produjeron cerca de 1,5 millones de Tm de PCB's en todo el mundo entre 1929 y 1979, hasta su prohibición en el Acta de Control de Sustancias Tóxicas (TSCA) (Habibullah-AlMamun, et al., 2019; Wong, et al., 2004; Xing et al., 2005).

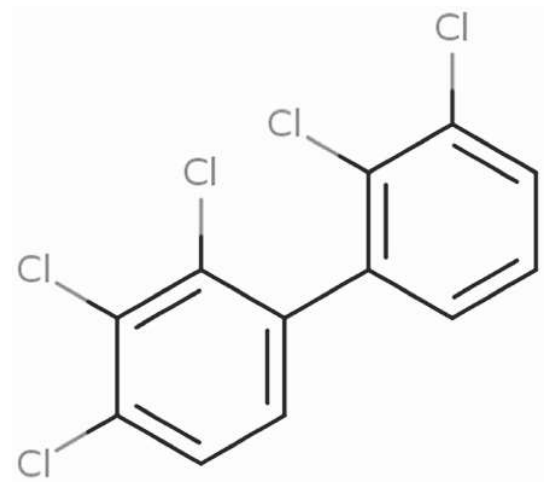

Figura 1. Estructura molecular del Aroclor 1254

Los equipos eléctricos obsoletos que operaron con aceites dieléctricos con PCB's, al ser desechados en vertederos comunes sin los controles y cuidados apropiados, son una fuente importante de contaminación. El aceite contaminado con PCB's puede derramarse en el suelo y llegar a cuerpos de agua subterráneas o su- 
perficiales ya sea accidentalmente o por corrosión y ruptura de los tanques que los almacenan (HabibullahAl-Mamun et al., 2019; Law \& Jepson, 2017). Una vez presentes en el agua, estos contaminantes pueden incorporarse a la cadena alimentaria y acumularse en organismos acuáticos, los cuales al ser consumidos por seres humanos Ilegan a causar graves daños a su salud (Atmaca et al., 2019; Nadarajan et al., 2017).

Aroclor 1254 es el nombre comercial de una mezcla de PCB's producidos por la corporación Monsanto, y su numeración es debido a que poseen 12 átomos de carbono y $54 \%$ en peso de átomos de cloro, su estructura se presenta en la Figura 1. Esta mezcla fue la segunda más producida en el mundo y es especialmente tóxica por sus propiedades similares a la de las dioxinas (Lopera \& Aguire, 2006; Parent et al., 2016). Sobre las posibilidades de eliminación de los PCB's, debe considerarse que estos son sustancias inertes, tóxicas y térmicamente estables, por tanto, su descomposición natural es poco pro- bable y los tratamientos convencionales son poco efectivos (Moreno, 2016; Law \& Jepson, 2017). La toxicidad de los PCB's sumada a la resistencia que tienen a la oxidación por microorganismos, hace que tratamientos biológicos sean inaplicables (Izquierdo \& Peña, 2016), aspecto que motiva la aplicación de métodos alternativos de tratamiento como son los procesos de oxidación avanzada (POA's).

Actualmente, el uso de equipos eléctricos que contienen aceites con PCB's está prohibido en Ecuador y los aún existentes son almacenados por el Ministerio de Energía y Recursos Naturales No Renovables a la espera de una solución viable para el tratamiento de estos aceites contaminados (Frías \& Gia, 2014).

En este trabajo se propone estudiar la transferencia de fase del Aroclor 1254 desde el aceite dieléctrico hacia una fase acuosa para su posterior degradación fotocatalítica empleado $\mathrm{TiO}_{2}$ irradiado con electrones acelerados. 


\section{MATERIALES Y MÉTODOS}

\section{Cuantificación de Aroclor 1254 en aceite dieléctrico y fase acuosa}

Para todas las pruebas se utilizó como estándar un aceite dieléctrico proporcionado por el Laboratorio de Análisis de Residuos de Contaminantes Orgánicos del Ministerio de Electricidad y Recursos Naturales. El Aroclor 1254 presente en el aceite dieléctrico se determinó mediante cromatografía de gases acorde al método ASTM D 4059. Las inyecciones se realizaron en un cromatógrafo de gases Modelo 6890 (Agilent) equipado con un automuestrador G4513A (Agilent) y un detector de captura de electrones (ECD). Se usó una columna para compuestos halogenados con composición $5 \%$ fenilpolimetilsiloxano $(30 \mathrm{~m}$ de largo, 0,25 mm de diámetro interno, 0,25 $\mu \mathrm{m}$ de espesor de film, Agilent HP-5). Para la construcción de la curva de calibración se prepararon soluciones de 0,$1 ; 0,25 ; 0,5 ; 1,0 ; 1,5$ y $2,0 \mathrm{mg}$ de Aroclor 1254/L de solvente, y se inyectaron al cromatógrafo por duplicado, posteriormente se inyectaron soluciones estándar de concentración conocida de Aroclor 1254 para comparar el resultado obtenido con el valor de concentración conocida. El software ChemStations versión A.08.03 fue utilizado para controlar la elución y registrar los cromatogramas.

\section{Transferencia de Aroclor 1254 a fase acuosa}

Se probaron dos alternativas para transferir las moléculas de Aroclor 1254 a la fase acuosa. La primera alternativa fue con Polietilenglicol 400 (PEG 400) debido a sus características anfipáticas capaces de atrapar las moléculas de Aroclor 1254 y Ilevarlas a la fase acuosa. A $25 \mathrm{~mL}$ de solución de PEG 400 al $10 \%$ (v/v), se agregaron $250 \mu \mathrm{L}$ de aceite dieléctrico usado y se mezcló con agitación a $120 \mathrm{rpm}$ durante $3 \mathrm{~h}$. La segunda alternativa fue empleando Tritón ${ }^{\circledR}$ X-100 aplicando el mismo procedimiento mencionado para el PEG 400. La cuantificación de Aroclor 1254 se realizó mediante la toma de alícuotas de las mezclas de aceite dieléctrico con los agentes de transferencia de fase (PEG 400 o Tritón® X-100) cada hora (tres alícuotas 
en total para cada alternativa). El contenido de Aroclor 1254 en el aceite tratado con PEG 400 o Tritón® X-100 fue cuantificado mediante cromatografía de gases, descrito previamente.

\section{Degradación de Aroclor 1254 por fotocatálisis heterogénea con $\mathrm{TiO}_{2}$ irradiado}

Para la degradación fotocatalítica de Aroclor 1254 se utilizó $\mathrm{TiO}_{2}$ rutílico microparticulado (Ti-Pure ${ }^{\circledR}$ R-902+, DuPont), previamente irradiado. La irradiación del $\mathrm{TiO}_{2}$ se realizó con un acelerador de electrones ELU-6U. Se colocó el $\mathrm{TiO}_{2}$ en bolsas transparentes de polietileno y se sometieron dichas bolsas a irradiación con dosis de 20 y 60 kGy. Luego de que el Aroclor 1254 ya estuvo en fase acuosa, ésta fue sometida al tratamiento por fotocatálisis heterogénea con $\mathrm{TiO}_{2}$ y radiación UV como fuente de energía. El estudio cinético de fotodegradación de Aroclor 1254 se realizó con $50 \mathrm{~mL}$ de la fase acuosa obtenida del tratamiento del aceite y $50 \mathrm{mg}$ de $\mathrm{TiO}_{2}$ (Wong et al., 2004).

El $\mathrm{TiO}_{2}$ fue dispersado en la fase acuosa por sonicación por 10 min mediante un baño ultrasónico
(CPXH, Branson). Luego la mezcla se sometió a radiación ultravioleta con una lámpara UV T8 de $15 \mathrm{~W}$ de potencia (Zhejiang Changhong). Se tomaron alícuotas de $1 \mathrm{~mL}$ cada 20 min durante las dos primeras horas $y$, posteriormente, una alícuota de $1 \mathrm{~mL}$ cada 60 min hasta completar las $4 \mathrm{~h}$ de tratamiento. Además, se realizaron ensayos con $\mathrm{TiO}_{2}$ (sin irradiar e irradiado) en presencia radiación UV y en ausencia de esta, con la finalidad de evaluar la influencia de la adsorción en la remoción de Aroclor 1254 del agua.

\section{Mineralización de Aroclor 1254}

El grado de mineralización se siguió mediante COT. Las mediciones de COT en las fases acuosas (tratadas y sin tratar) se realizaron mediante el Método Estándar 9060 (American Public Health Asosciation [A.P.H.A], 1995). Las mediciones de COT se llevaron a cabo con un analizador de Carbono Orgánico Total TOC-5000A. Dado que el agente de transferencia es un compuesto orgánico, se espera de este un aporte al contenido de COT de la fase acuosa resultante del tratamiento del aceite dieléctrico. Es decir, el contenido de COT en la fase 
acuosa después de haber transferido el Aroclor 1254 del aceite a la fase acuosa es, esencialmente, la suma del carbono orgánico aportado por el agente de transferencia y el Aroclor 1254.

\section{RESULTADOS}

\section{Transferencia de Aroclor 1254 a la fase acuosa}

En la Tabla 1, se presenta la transferencia porcentual de moléculas de Aroclor 1254 alcanzada al añadir $250 \mu \mathrm{L}$ de aceite dieléctrico a $25 \mathrm{~mL}$ de soluciones de PEG 400 y Tritón X100 al $10 \% \mathrm{v} / \mathrm{v}$, respectivamente, después de mantenerlas en agitación constante a 120 rpm durante 3 horas. Como se puede evidenciar en la Tabla 1, el porcentaje de transferencia de fase oleosa a fase acuosa del Aroclor 1254, es mucho mayor cuando se emplea Triton X-100, por lo que los estudios de degradación se precederán con este agente de transferencia.
Tabla 1. Porcentaje de transferencia de Aroclor 1254 a la fase acuosa en función del tiempo de agitación

\begin{tabular}{ccccc}
\hline & $\begin{array}{c}\text { Concentración } \\
\text { Aroclor 1254 en } \\
\text { la fase acuosa } \\
\text { (mg/L) }\end{array}$ & \multicolumn{2}{c}{$\begin{array}{c}\text { Transferencia } \\
\text { de Aroclor 1254 } \\
\text { a la fase acuosa } \\
(\%)\end{array}$} \\
\hline $\begin{array}{c}\text { Tiempo } \\
\text { (h) }\end{array}$ & $\begin{array}{c}\text { PEG } \\
\mathbf{4 0 0}\end{array}$ & $\begin{array}{c}\text { Tritón } \\
\text { X-100 }\end{array}$ & $\begin{array}{c}\text { PEG } \\
\mathbf{4 0 0}\end{array}$ & $\begin{array}{c}\text { Tritón } \\
\text { X-100 }\end{array}$ \\
\hline 1 & 0,47 & 85,2 & 0,42 & 75,35 \\
2 & 0,14 & 103,6 & 0,12 & 91,62 \\
3 & 0,38 & 110,8 & 0,34 & 97,99 \\
\hline
\end{tabular}

Nota: La concentración inicial de Aroclor 1254 en el aceite dieléctrico usado en estos ensayos fue $11.307,0 \mathrm{mg} / \mathrm{L}$.

\section{Fotodegradación de Aroclor 1254 con $\mathrm{TiO}_{2}$ irradiado}

Utilizando Tritón X-100 como el agente de transferencia de fase, se evaluó la degradación fotocatalítica de Aroclor 1254 con $\mathrm{TiO}_{2}$ sin irradiar e irradiado, dosis de 20 y 60 kGy, (Figura 2). Para los ensayos de degradación, se usaron $50 \mathrm{mg}$ de $\mathrm{TiO}_{2}$ por cada $50 \mathrm{~mL}$ de fase acuosa a tratar, equivalente a una concentración de 
100 mg/L (Wong et al., 2004), y esta cantidad se mantuvo constante para todos los ensayos.
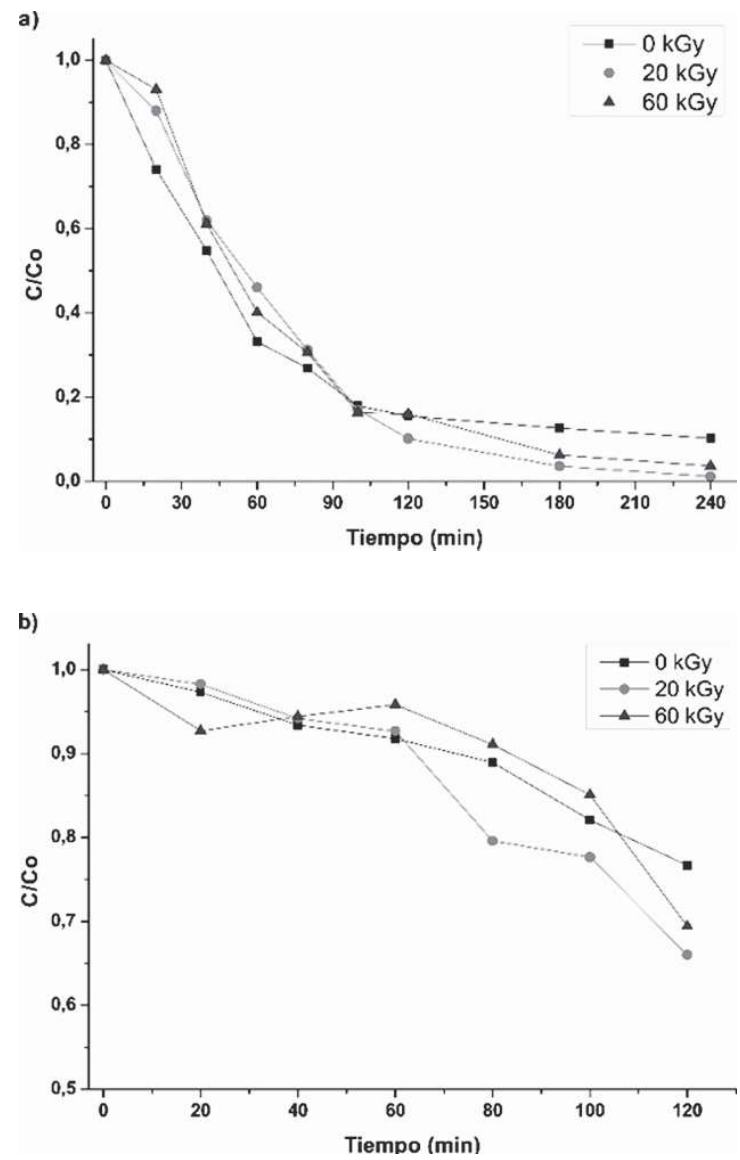

Figura 2. a) Degradación fotocatalítica de Aroclor 1254 en fase acuosa y b) Adsorción de Aroclor 1254 en $\mathrm{TiO}_{2}$ sin irradiar e irradiado (20 kGy y $60 \mathrm{kG}$ )

En la Tabla 2 se presentan las constantes de velocidad de pseudo primer orden para las reacciones de degradación fotocatalítica de Aroclor 1254. Además, también se calcularon los tiempos de vida media del Aroclor 1254, en cada caso.
Tabla 2. Constante de velocidad, tiempos de vida media $\left(t_{1 / 2}\right)$ y porcentaje de degradación para el Aroclor 1254, de acuerdo a los tratamientos realizados al $\mathrm{TiO}_{2}$ utilizado

\begin{tabular}{lccc}
\hline TiO $_{2}$ utilizado & $\mathrm{k}\left(\mathrm{min}^{-1}\right)$ & $\begin{array}{c}\mathrm{t}_{1 / 2} \\
(\mathrm{~min})\end{array}$ & $\begin{array}{c}\text { Degradación } \\
\text { Aroclor 1254 } \\
(\%)\end{array}$ \\
\hline Sin irradiar 0 & 0,0163 & 43 & 89,46 \\
Irradiado (20 kGy) & 0,0193 & 36 & 98,36 \\
Irradiado (60 kGy) & 0,0173 & 40 & 94,43 \\
\hline
\end{tabular}

\section{Mineralización de Aroclor 1254}

En la Figura 3 se muestra el COT luego de la fotodegradación, con $\mathrm{TiO} 2$ irradiado a diferentes dosis. Además, se muestra un estudio de la cantidad de COT que aporta cada compuesto presente en la muestra (Triton X-100 y Aroclor).
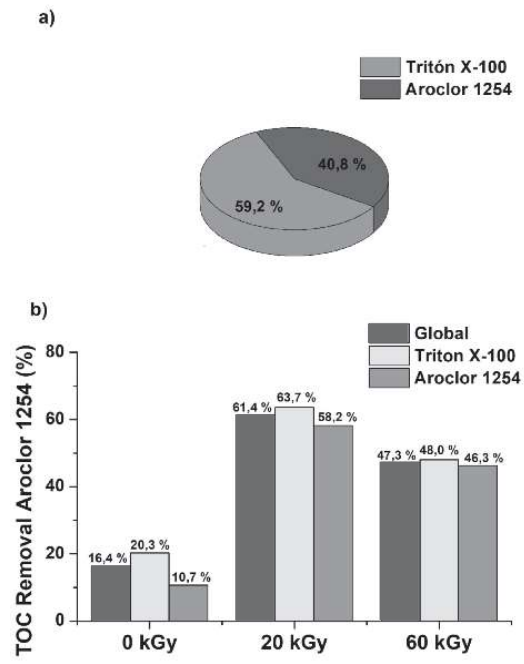

Figura 3. Porcentajes de remoción de COT mediante fotodegradación con $\mathrm{TiO}_{2}$ sin irradiar (0 kGy) e irradiado (20 kGy y $60 \mathrm{kGy}$ ): a) aporte de COT del Tritón X-100 y Aroclor 1254, b) COT global y de cada uno de los aportantes 


\section{DISCUSIÓN}

Transferencia de Aroclor 1254 a la fase acuosa

En la Tabla 1, se evidencia que a pesar de las características anfipáticas del PEG 400 y de haber sido exitosamente utilizado en el pasado para transferir PCB's desde moluscos contaminados hacia disoluciones acuosas (Nadarajan et al., 2017), la eficiencia no es la misma cuando se busca transferir los PCB's de aceite dieléctrico a un solvente polar como el agua. La eficiencia en la transferencia del Aroclor 1254 de la fase oleosa a la fase acuosa con el Tritón X-100 es mucho mayor gracias a la formación de micelas. Se generan estructuras de forma esférica en las que los grupos funcionales hidrofílicos se ubican en la parte externa, mientras que, la parte hidrofóbica de las moléculas se orienta hacia el centro de la esfera, donde quedan atrapadas las moléculas hidrofóbicas de PCB's (Fröschl et al., 1997). Por esta razón, se seleccionó el Tritón X-100 como agente de transferencia de fase para los estudios de degradación del Aroclor 1254. Una explicación teórica ampliada se publicará más adelante incluyendo estudios que respalden esta afirmación.

\section{Degradación de Aroclor 1254 por fotocatálisis heterogénea con dió- xido de titanio irradiado}

La Figura 2a muestra la fotodegradación de Aroclor 1254 en fase acuosa con $\mathrm{TiO}_{2}$ sin irradiar (0 kGy) e irradiado (20 y 60 kGy). La degradación del Aroclor 1254 sobre los tres tipos de $\mathrm{TiO}_{2}$ (de acuerdo a los tratamientos realizados) tienen un comportamiento similar hasta aproximadamente 100 min. Las curvas muestran que después de 240 min, el $\mathrm{TiO}_{2}$ irradiado a 20 kGy permite alcanzar una mayor remoción del Aroclor 1254. Por otro lado, la Figura $2 \mathrm{~b}$ muestra la adsorción del Aroclor 1254 en función del tiempo, en ausencia de luz. Con este experimento se pudo confirmar que el $\mathrm{TiO}_{2}$ irradiado a 20 kGy adsorbe más Aroclor 1254 respecto de los otros $\mathrm{TiO}_{2}$. Como consecuencia de la irradiación, la superficie del $\mathrm{TiO}_{2}$ sufre modificaciones superficiales, ocasionando que el comportamiento adsortivo del $\mathrm{TiO}_{2}$ cambie dependiendo de la dosis irradiada (Cropper, 1962). En 
este trabajo para el caso del $\mathrm{TiO}_{2}$ irradiado a 20 kGy se alcanza la mayor degradación del Aroclor 1254, lo cual se asocia a la mayor adsorción encontrada en el $\mathrm{TiO}_{2}$ irradiado a esta dosis (resultados preliminares). Por lo que, se puede concluir preliminarmente que al someter a diferentes dosis de irradiación un material semiconductor, este adquiere propiedades adsortivas particulares, que pueden favorecer la adsorción de determinadas moléculas; y esto a su vez promover una mejor transferencia de masa hacia los sitios activos del fotocatalizador, provocando una degradación más rápida de los contaminantes.

Estudios realizados sobre el efecto de radiación gama en fotocatálisis con $\mathrm{TiO}_{2}$, han reportado que la aplicación de radiación gama con dosis cercanas a 28 kGy incrementan la actividad fotocatalítica del $\mathrm{TiO}_{2}$ debido a la aparición de "defectos" superficiales generados por la radiación, cuyo tipo y concentración es proporcional a la dosis de irradiación aplicada en el fotocatalizador (Kralchevska et al., 2012).
Otro estudio similar concluye que la aplicación de radiación en nanotubos de $\mathrm{TiO}_{2}$ incrementa su actividad fotocatalítica por el aumento de la generación de radicales hidroxilos $\left({ }^{\circ} \mathrm{OH}\right)$ (Zhang et al., 2017). Estos datos apoyan la interpretación de los resultados representados en la Figura 3, y a pesar de que la fuente de irradiación es diferente, se pudo lograr un efecto similar si se bombardea con electrones acelerados en dosis equivalentes (Chitose et al., 2003).

En la Tabla 2 se presentan las constantes de velocidad de pseudo primer orden para la degradación fotocatalítica de Aroclor 1254 con el $\mathrm{TiO}_{2}$ sin irradiar e irradiado. Se corrobora con estos resultados, que la degradación en el $\mathrm{TiO}_{2}$ irradiado es más rápido que el no irradiado.

\section{Mineralización de Aroclor 1254}

Se pudo determinar el aporte del Tritón X-100 y el Aroclor 1254 al COT global. Se encontró que el Tritón X100 aporta con un 59,2 \% al COT global mientras que el Aroclor 1254 con el restante 40,8\%, Figura 3 a. 
Después del tratamiento fotocatalítico con el $\mathrm{TiO}_{2}$ sin irradiar e irradiado, nuevamente se determinó el contenido de COT para identificar cual $\mathrm{TiO}_{2}$ permite una mayor mineralización.

Los resultados mostrados en la Figura $3 \mathrm{~b}$, indican que sobre $\mathrm{TiO}_{2}$ sin irradiar se obtuvo la remoción de COT mayor, tanto del COT global como de los aportantes al COT individual. Con el $\mathrm{TiO}_{2}$ irradiado solo se logra mineralizar 10,7 \% del Aroclor 1254 en 240 min de reacción. Para tiempos iguales de reacción, cuando se utilizó el $\mathrm{TiO}_{2}$ irradiado, se alcanzaron mayores remociones de COT, y fue con el $\mathrm{TiO}_{2}$ irradiado con dosis de $20 \mathrm{kGy}$ que se logró la mayor remoción de COT asociada al Aroclor 1254 (58,2 $\%$ ). Es posible observar mayor mineralización de Aroclor 1254 cuando se utilizó $\mathrm{TiO}_{2}$ irradiado; resultado igualmente destacado por Chitose et al. (2003), quienes reportaron la mejora en la mineralización de la materia orgánica cuando el fotocatalizador es irradiado.

\section{CONCLUSIONES}

Se obtuvo que el mejor agente de transferencia de fase para el Aroclor 1254, en altas concentraciones, desde el aceite dieléctrico hacia la fase acuosa es el Tritón X-100.

Respecto al fotocatalizador utilizado, tanto del $\mathrm{TiO}_{2}$ sin irradiar (0 kGy) como del irradiado (20 y 60 kGy), permitió la degradación de Aroclor 1254 en fase acuosa. Sin embargo, con el $\mathrm{TiO}_{2}$ irradiado se logró una mayor degradación y mineralización, comparado con el $\mathrm{TiO}_{2}$ sin irradiar. Luego de 240 min de reacción, con el $\mathrm{TiO}_{2}$ irradiado a la dosis de 20 kGy se logró la mayor degradación (98,36 \%) y mineralización del Aroclor 1254 $(63,7$ \% de remoción del COT debido al Aroclor 1254). Se confirmó lo sugerido en estudios previos: el uso de radiaciones ionizantes, en este caso electrones acelerados, mejora las características fotocatalíticas del $\mathrm{TiO}_{2}$. 


\section{LISTA DE REFERENCIAS}

American Public Health Association, A. P. H. A. (1995). Standard methods for the examination of water and wastewater. Washington, DC: American Public Health Association. (Vol. 21).

Atmaca, E., Das, Y. K., Yavuz, O., \& Aksoy, A. (2019). An evaluation of the levels of organochlorine compounds (OCPs and PCBs) in cultured freshwater and wild sea fish eggs as an exposure biomarker for environmental contamination. Environmental Science and Pollution Research, 26(7), 7005-7012. https://doi.org/10. 1007/s11356-019-04207-0

Camargo Moreno, C. E. (2014). Biotratamiento de Aceites Dieléctricos Usados (tesis de maestría). Universidad Nacional de Colombia. Obtenido de: http://bdigital.unal. edu.co/57173/7/CarlosE.CamargoMoreno.2016.pdf

Chitose, N., Ueta, S., Seino, S., \& Yamamoto, T. A. (2003). Radiolysis of aqueous phenol solutions with nanoparticles. 1. Phenol degradation and TOC removal in solutions containing TiO2 induced by UV, $\sqsubset$-ray and electron beams. Chemosphere, 50(8), 1007-1013. https://doi.org/10.1016/S0045-6535(02)00642-2

Cropper, W. H. (1962). Radiation Effects on the Reactivity of Solid Surfaces: Defects produced in solids by radiation fields can enter into chemical reactions on the surface. Science, 137(3534), 955-961. https://doi.org/10.1126/science.137.3534.955

Frías, M. M., \& Gia, J. (2014). Descomposición del Bifenilo Policlorado (PCB) Aroclor 1260 en aceites dieléctricos, por radiólisis del agua, inducida por radiación gamma del isótopo de cobalto 60. Universidad Central Del Ecuador. Obtenido de: http://www.dspace.uce.edu.ec/handle/25000/6284

Fröschl, B., Stangl, G., \& Niessner, R. (1997). Combination of micellar extraction and GC-ECD for the determination of polychlorinated biphenyls (PCBs) in water. Fresenius' Journal of Analytical Chemistry, 357(6), 743-746. https://doi.org/10.1007/ s002160050241 
Habibullah-Al-Mamun, M., Ahmed, M. K., Islam, M. S., Tokumura, M., \& Masunaga, S. (2019). Occurrence, distribution and possible sources of polychlorinated biphenyls (PCBs) in the surface water from the Bay of Bengal coast of Bangladesh. Ecotoxicology and environmental safety, 167, 450-458. https://doi.org/10.1016/j.ecoenv. 2018.10.052

Huang, Q., \& Hong, C.-S. (2000). $\mathrm{TiO}_{2}$ photocatalytic degradation of PCBs in soil-water systems containing fluoro surfactant. Chemosphere, 41(6), 871-879. https://doi. org/10.1016/S0045-6535(99)00492-0

Izquierdo Condoy, M., \& Peña Pontón, E. (2016). Diseño de sistemas de biodegradación aerobia y anaerobia a escala piloto para la descontaminación de aceites dieléctricos con bifenilos policlorados (PCB's) (tesis de pregrado). Escuela Politécnica Nacional. Obtenido de: https://bibdigital.epn.edu.ec/handle/15000/16557

Kralchevska, R., Milanova, M., Tsvetkov, M., Dimitrov, D., \& Todorovsky, D. (2012). Influence of gamma-irradiation on the photocatalytic activity of Degussa P25 $\mathrm{TiO}_{2}$. Journal of Materials Science, 47(12), 4936-4945. https://doi.org/10.1007/s10853012-6368-4

Law, R. J., \& Jepson, P. D. (2017). Europe's insufficient pollutant remediation. Science, 356(6334), 148.

Lopera Posada, E., \& Aguire Cardona, J. (2006). Purification of mineral insulating oil contaminated with polychlorinated biphenyls (PCB's). Dyna, 73(150), 75-88.

Nadarajan, R., Bakar, W. A. W. A., Ali, R., \& Ismail, R. (2017). Method for polychlorinated biphenyls removal from mussels and its photocatalytic dechlorination. Applied Catalysis B: Environmental, 218, 327-337. https://doi.org/10.1016/j.apcatb. 2017.06.066

Parent, A. S., Pinson, A., Woods, N., Chatzi, C., Vaaga, C. E., Bensen, A., Westbrook, G. L. (2016). Early exposure to Aroclor 1254 in vivo disrupts the functional synaptic development of newborn hippocampal granule cells. European Journal of Neuroscience, 44(12), 3001-3010. https://doi.org/10.1111/ejn.13437 
Wong, K. H., Tao, S., Dawson, R., \& Wong, P. K. (2004). Optimization of photocatalytic oxidation of 2,2',3,3' - tetrachlorobiphenyl. Journal of Hazardous Materials, 109(13), 149-155. https://doi.org/10.1016/j.jhazmat.2004.03.006

Xing, Y., Lu, Y., Dawson, R. W., Shi, Y., Zhang, H., Wang, T., Ren, H. (2005). A spatial temporal assessment of pollution from PCBs in China. Chemosphere, 60(6 SPEC. ISS.), 731-739. https://doi.org/10.1016/j.chemosphere.2005.05.001

Zhang, Q., Ye, S., Chen, X., Song, X., Li, L., \& Huang, X. (2017). Photocatalytic degradation of ethylene using titanium dioxide nanotube arrays with $\mathrm{Ag}$ and reduced graphene oxide irradiated by $\gamma$-ray radiolysis. Applied Catalysis B: Environmental, 203, 673-683. https://doi.org/10.1016/j.apcatb.2016.10.034 